\title{
Characterization of absorption loss for UWB body tissue propagation model
}

\begin{abstract}
The paper presents the results of absorption loss for body tissue propagation model which is characterized by the specific absorption rate (SAR) investigated on heterogeneous normal and tumour affected tissues of numerical human breast models. The absorption loss is evaluated using UWB signals of 4 to $8 \mathrm{GHz}$. The analysis of the study shows that the absorption loss in the body tissue models increases with frequency and varies with the change in dielectric properties of body tissue models. Deviation of absorption loss up to $0.9 \mathrm{~dB}$ is observed in tumour affected model compared to normal model.

Keyword: Specific absorption rate (SAR); Heterogeneous normal and tumour affected tissue models; Ultra wideband (UWB); Finite integration technique (FIT); Body tissue propagation model
\end{abstract}

